\title{
Salivary Carcinoembryonic Antigen (CEA) in Cystic Fibrosis
}

\author{
J. M. MACSWEEN AND C. T. GILLESPIE \\ Departments of Medicine and Paediatrics, Dalhousie University and Camp Hill Hospital, Halifax, \\ Nova Scotia, Canada
}

\begin{abstract}
Summary
Increased concentrations of carcinoembryonic antigen (CEA) have been reported in the sera and sputum of patients with cystic fibrosis. We have found significantly higher concentrations of CEA in both extracted and unextracted saliva of patients with cystic fibrosis compared to age-matched controls and a comparable group of patients with bronchial asthma. The mean CEA concentrations in saliva were also somewhat higher in patients with more severe respiratory disease. The concentration of CEA in the saliva of obligate heterozygotes was similar to healthy controls. This suggests that increased salivary CEA in patients with cystic fibrosis relates to the expression of the disease rather than the genetic predisposition.
\end{abstract}

\section{Speculation}

Increased salivary carcinoembyronic antigen (CEA) in cystic fibrosis may reflect primary changes in the secretion of glycoproteins by exocrine glands. This could result in abnormal properties of these secretions.

Carcinoembryonic antigen is a glycoprotein originally identified in tumors of the gastrointestinal tract and fetal tissue $(7,8)$. Antigenically similar material has been detected in a variety of tissues and biological fluids both in the presence and absence of malignancies $(5,9,11,14,15)$. The biochemical identity of these substances with tumor-associated CEA is open to question, but they are recognized as such in radioimmunoassays because of their antigenic similarity or identity.

Martin and Devant (13) reported the presence of CEA in normal human saliva, and Davidson et al. (3) reported that the concentration of CEA in the serum of patients with cystic fibrosis was increased over controls. This was confirmed by Wilson et al. (17). These observations raised the question of whether the concentration of CEA in exocrine secretions was increased in this condition. Wu et al. (18) reported increased concentrations of CEA in the sputum as well as in the serum of patients with cystic fibrosis. The CEA concentration in the saliva of these patients was higher than that of controls but the difference was not statistically significant. Therefore, the observed increased CEA could be secondary to inflammatory changes in sputum-producing respiratory mucosa. We measured the salivary concentration of CEA to determine if increased CEA production in cystic fibrosis represents a more basic abnormality in exocrine secretion.

\section{MATERIALS AND METHODS}

\section{PATIENTS}

The diagnosis of cystic fibrosis was established on the basis of elevation of sweat chloride and typical clinical course. Asthmatic controls had clinical and laboratory evidence of chronic airway obstruction. Healthy age-matched controls were selected from offspring of laboratory personnel and children in the hospital for minor surgery procedures. The mothers of patients with cystic fibrosis were tested as obligate heterozygotes of the cystic fibrosis gene and compared to healthy females in the same age range.

\section{EXTRACTION}

Saliva specimens were collected in clean plastic receptacles and were processed immediately or stored at $-20^{\circ} \mathrm{C}$. Extraction of specimens with perchloric acid was carried out by adding $100 \mu \mathrm{l}$ $4 \mathrm{M}$ perchloric acid to $300 \mu \mathrm{l}$ saliva with constant agitation on a vortex mixer. The tubes were centrifuged at $5000 \times \mathrm{g}$, and the supernatant fluid was dialyzed overnight against four changes of $0.014 \mathrm{M}$ phosphate-buffered saline, $\mathrm{pH} 7.4\left(\mathrm{Na}_{2} \mathrm{HPO}_{4}, 0.008 \mathrm{M}\right.$ $\mathrm{NaH}_{2} \mathrm{PO}_{4}, 0.0015 \mathrm{M}-\mathrm{NaCl}, 0.131 \mathrm{M}$ ). The dialyzed extract was assayed for CEA content immediately or stored at $-20^{\circ} \mathrm{C}$. Protein in both extracted and unextracted saliva was measured by the technique of Lowry et al. (10) using bovine serum albumin as a standard. The limit of reliable sensitivity was $50 \mu \mathrm{g}$.

\section{RADIOIMMUNOASSAY FOR CEA}

Both extracted and nonextracted specimens were assayed in duplicate for CEA by a micro method described previously (12) using reagents obtained commercially from Hoffman-La Roche Co., (Vaudreuil, Quebec, Canada). Goat antiserum to CEA as well as ${ }^{125} \mathrm{I}$-labeled CEA were diluted in $0.01 \mathrm{M}$ Tris buffer, $\mathrm{pH}$ 7.5 , containing $3 \%$ bovine serum albumin. Twenty-five microliters of anti-CEA were added to the same volume of extracted or unextracted saliva or doubling dilutions of a standard solution of unlabeled CEA. Fifty microliters of ${ }^{125} \mathrm{I}$-labeled CEA were added to each tube. After mixing, the tubes were held at $37^{\circ} \mathrm{C}$ for $2 \mathrm{hr}$ and overnight at $4^{\circ} \mathrm{C}$. One hundred microliters of a saturated solution of ammonium sulfate were added to each tube with immediate mixing. After $30 \mathrm{~min}$ at $4^{\circ} \mathrm{C}$, the tubes were centrifuged at $5000 \times \mathrm{g}$, and $100 \mu \mathrm{l}$ of supernatant fluid were removed from each tube. The radioactivity in each sample was determined in a gamma spectrometer.

\section{COLUMN CHROMATOGRAPHY}

Extracted and unextracted aliquots of saliva $(100 \mu l)$ were applied to a $1.5 \times 30 \mathrm{~cm}$ Bio-Gel P-200 column equilibrated with $0.14 \mathrm{M}$ phosphate-buffered saline, $\mathrm{pH} 7.4$, containing $0.1 \%$ bovine serum albumin. The column was calibrated with blue dextran, (Pharmacia Fine Chemicals, Piscataway, N. Y.) carrier-free ${ }^{125} \mathrm{I}$, ${ }_{125}$ I-labeled ribonuclease (Sigma Chemical Co., St. Louis, MO.), and purified ${ }^{125}$ I-labeled CEA (Hoffman-La Roche, Inc., Nutley, N. J.). One $\mathrm{ml}$ fractions were collected from the column at $4^{\circ} \mathrm{C}$ and assayed for CEA content as described above.

\section{RESULTS}

CEA was measured in the saliva of 12 patients with cystic fibrosis, 9 age-matched healthy controls, and 8 patients with bronchial asthma (Table 1). CEA was measured directly and also after extraction with perchloric acid. Protein was not detectable by the technique of Lowry (10) in the saliva of the healthy controls after perchloric acid extraction. Therefore, CEA concentration 
was only expressed in $\mathrm{ng} / \mathrm{ml}$ in this group. In all cases, the mean CEA concentration was highest in the patients with cystic fibrosis, lowest in the healthy controls, and intermediate in patients with asthma. The following differences were statistically significant by the unpaired $t$ test: unextracted saliva (CEA, ng/ml), cystic fibrosis greater than normal controls $(P<0.05)$; extracted saliva (CEA. $\mathrm{ng} / \mathrm{ml})$, cystic fibrosis greater than asthmatics $(P<0.05)$, cystic fibrosis greater than normal controls $(P<0.02)$; extracted saliva (CEA, ng/mg protein): cystic fibrosis greater than asthmatics $(P$ $<0.02$ ). There was no significant difference between the concentration of CEA in the saliva of the obligate heterozygotes and the adult controls (Table 1).

The severity of disease in the patients with cystic fibrosis was estimated by an observer who was unaware of the CEA levels and documented on the basis of the modified Schwachman-Kulcychi system (4). The severity of pulmonary involvement was also assessed on the basis of chest X-ray scores (4) (a score of less than 19 suggesting irreversible disease and a score of 25 indicating a normal X-ray). The patients with more severe respiratory disease on the basis of chest X-ray scores had somewhat higher levels of salivary CEA than did those with milder disease (Table 2). However, the differences were not statistically significant due to considerable variability in the CEA concentrations. There were no consistent differences in salivary CEA relating to the overall clinical status of the patients.

One patient with asthma had a very high concentration of salivary CEA when measured directly $(4000 \mathrm{ng} / \mathrm{ml})$ which was markedly reduced after extraction with perchloric acid (50 ng/ $\mathrm{ml}$ ). This anomaly was investigated further by adding aliquots of the saliva to a Bio-Gel P-200 column and measuring the CEA content of fractions eluted after column chromatography. The results are shown in Figure 1. There was an initial peak of CEA activity near the excluded volume approximating the peak of radiolabeled purified CEA. A second major peak was demonstrated near the included volume (molecular weight less than 13,700 ). The second peak was not seen when the saliva was extracted with perchloric acid.

\section{DISCUSSION}

CEA is a perchloric acid-soluble glycoprotein associated with neoplastic and fetal tissue. Extraction with perchloric acid has been found useful in recovering CEA from solutions containing other proteins $(1,9)$. Antigenically similar molecules have been detected in nonneoplastic tissue and exocrine secretions including saliva $(5,9,11,13-15)$. Their relationship to tumor-associated CEA is still unclear, although several molecular species have been identified and characterized $(1,2,6,16)$.

Increased concentrations of CEA have been reported in the sera and sputum of patients with cystic fibrosis, a disorder characterized by abnormal exocrine secretions (18). It was not clear whether increased CEA concentrations in the bronchial mucus was a primary phenomenon or secondary to repeated infections and inflammatory changes. Wu et al. (18) found that the concentrations of CEA in unextracted saliva of these patients were slightly higher than controls, but the difference was not statistically significant. After column chromatography, there were two peaks with CEA activity, with molecular weights of $>1,000,000$ and 200,000 . The latter corresponds to CEA extracted from human tumors.

We have found significantly higher concentrations of CEA in both extracted and unextracted saliva of patients with cystic fibrosis compared to age-matched controls and a comparable group of patients with bronchial asthma.

The mean concentration of CEA in unextracted saliva expressed as $\mathrm{ng} / \mathrm{mg}$ of protein in both patients and controls in our series was somewhat higher than that reported by $\mathrm{Wu}$ et al. (18). Differences in our results may reflect sampling differences because there was considerable variation between individuals or somewhat different specificities of the antisera because the reagents have not been standardized.

Salivary CEA in our series did not correlate closely with the severity of disease, suggesting that a relationship, if present, is an indirect one. Wu et al. (18) found a close correlation between plasma CEA and disease activity as well as sputum production, suggesting that elevated plasma CEA was largely derived from sputum. However, Wilson et al. (17) have reported elevated levels of plasma CEA in obligate heterozygotes. We have found elevated salivary CEA in patients with cystic fibrosis but not in the heterozygotes. On the basis of these studies, one may postulate that the genetic predisposition to cystic fibrosis is assocated with a systemic alteration in glycoprotein metabolism. This alteration is not reflected in exocrine secretions unless the disease is expressed. In specific terms, increased CEA in secretions is probably a marker associated with changes in other constituents rather than a direct determinant of disease activity. However, with the development of inflammatory changes in the bronchial mucosa, CEA production is stimulated as a secondary phenomenon, and this contributes

Table 1. CEA concentration in saliva

\begin{tabular}{|c|c|c|c|c|c|}
\hline \multirow[b]{2}{*}{ Group } & \multirow[b]{2}{*}{ No. } & \multicolumn{2}{|c|}{ Unextracted saliva } & \multicolumn{2}{|c|}{ Saliva extracted with perchloric acid } \\
\hline & & $\mathrm{CEA}(\mathrm{ng} / \mathrm{ml})$ & $\mathrm{CEA}$ (ng/mg protein) & $\mathrm{CEA}(\mathrm{ng} / \mathrm{ml})$ & CEA (ng/mg protein) \\
\hline Cystic fibrosis & 12 & $1321 \pm 355^{1}$ & $223 \pm 34.3$ & $250 \pm 41.8$ & $177 \pm 27.3$ \\
\hline Asthma & 8 & $655 \pm 479$ & $157 \pm 88.2$ & $102 \pm 36.8$ & $76 \pm 14.8$ \\
\hline Age-matched controls & 9 & $367 \pm 166$ & $139 \pm 49.8$ & $79 \pm 36.8$ & \\
\hline Obligate heterozygotes & 12 & $438 \pm 158$ & $56 \pm 16$ & $73 \pm 20$ & $65 \pm 21$ \\
\hline Adult controls & 11 & $363 \pm 122$ & $151 \pm 77$ & $71 \pm 30$ & $88 \pm 21$ \\
\hline
\end{tabular}

\footnotetext{
${ }^{1}$ Mean \pm S.E.
}

Table 2. The relationship of salivary CEA to the severity of disease in patients with cystic fibrosis

\begin{tabular}{|c|c|c|c|c|c|}
\hline \multirow[b]{2}{*}{ Severity of disease } & \multirow[b]{2}{*}{ No. } & \multicolumn{2}{|c|}{$\mathrm{CEA}(\mathrm{ng} / \mathrm{ml})$} & \multicolumn{2}{|c|}{ CEA (ng/mg protein) } \\
\hline & & Unextracted & PCA extract & Unextracted & PCA extract \\
\hline Severe (clinical score $50-80)^{1}$ & 7 & $1454 \pm 578^{2}$ & $307 \pm 63$ & $236 \pm 58$ & $177 \pm 44$ \\
\hline Mild (clinical score 81-100) & 5 & $1275 \pm 379$ & $269 \pm 61$ & $221 \pm 44$ & $240 \pm 78$ \\
\hline$x$-ray score less than 19 & 7 & $1839 \pm 519$ & $299 \pm 47$ & $277 \pm 51$ & $194 \pm 42$ \\
\hline$x$-ray score 19 and over & 5 & $735 \pm 290$ & $274 \pm 77$ & $164 \pm 41$ & $153 \pm 30$ \\
\hline
\end{tabular}

' Overall clinical score on the basis of the modified Schwachman-Kulcychi scoring system.

${ }^{2}$ Mean \pm S.E. 


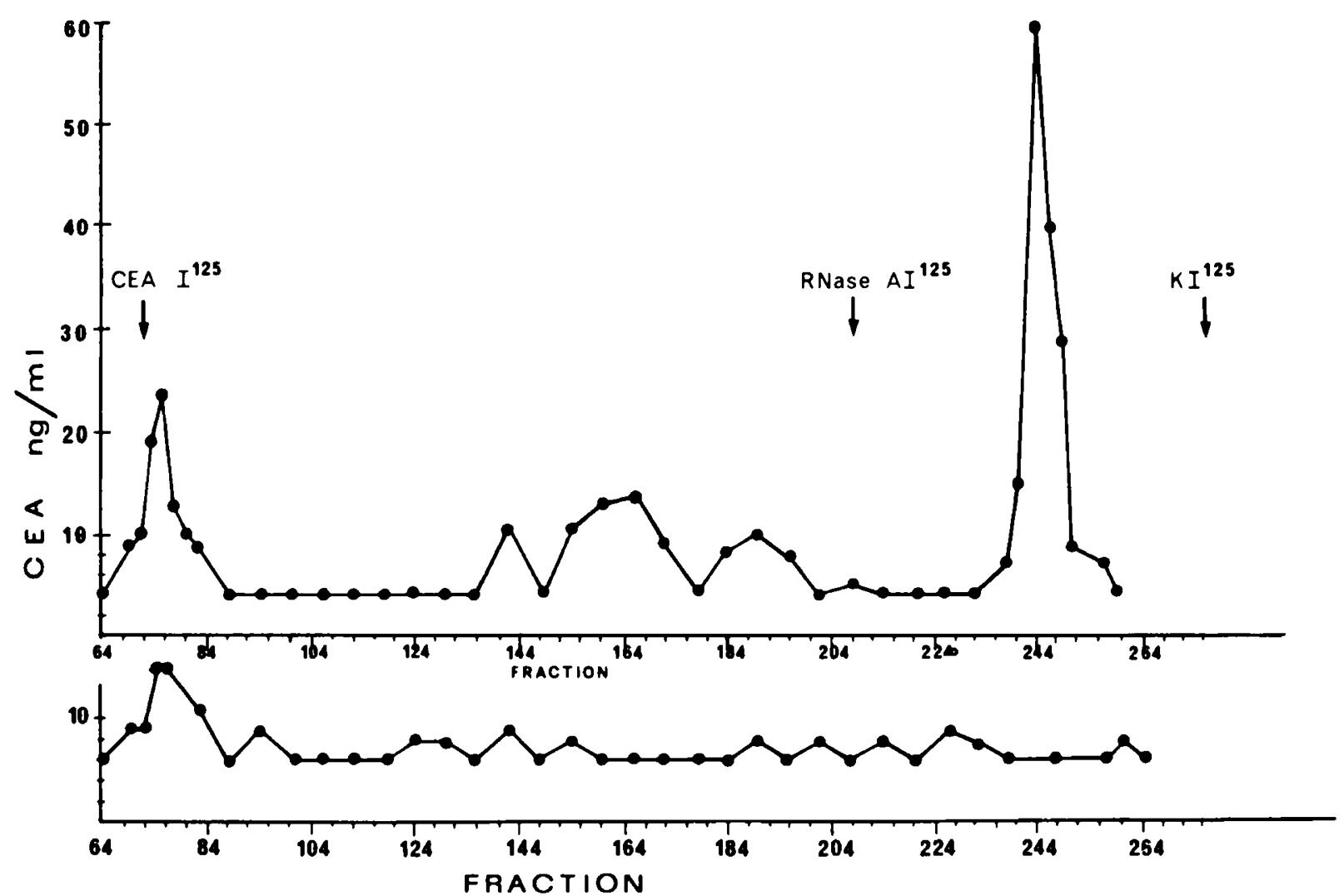

Fig. 1. CEA concentrations in eluted fractions from a Bio-Gel P-200 column. Upper panel, unextracted saliva; lower panel, saliva extracted with perchloric acid.

considerably to the plasma CEA which therefore reflects disease activity.

The CEA concentration in the saliva of children with asthma in our series was intermediate between cystics and healthy controls. This was largely due to a very high concentration of CEA in the unextracted saliva of a single individual. This patient had a history of recurrent infections as well as poor growth. The CEA appeared to be unusual in that it was markedly reduced after extraction with perchloric acid. We therefore went on to characterize the CEA by column chromatography. This showed that a large proportion of the CEA as measured by radio-immunoassay was of less than 13,700 molecular weight. This is clearly a different species than that found in tumors $(7,8)$ or in the saliva of patients with cystic fibrosis (18). The disappearance of this fraction after extraction by perchloric acid may be due to insolubility in perchloric acid or loss due to dialysis.

\section{REFERENCES AND NOTES}

1. Coligan. J. E.. Henkart, P. A., Todd. C. W., and Terry, W. D.: Heterogeneity of the carcinoembryonic antigen. Immunochemistry, 10: 591 (1973).

2. Darcy. D. A., Turberville, C.. and James, R.: Immunological study of carcinoembryonic action (CEA) and a related glycoprotein. Br. J. Cancer. 28: 147 (1973).

3. Davidson, A. G. F., Mincey. E. K., Israels. S., and Wilcox. M. E.: Carcinoembryonic antigen in cystic fibrosis. Lancet, 2: 1393 (1973).

4. Doershuk. C. F.. Matthews. L. W.. Tucker. A. S.. Nudelman. H.. Eddy, G., Wise. M., and Spector. S.: A five year clinical evaluation of a therapeutic program for patients with cystic fibrosis. J. Pediatr.. 65: 677 (1964).

5. Dyce. B. J., and Haverback. B. J.: Free and bound carcinoembronic antigen in neoplasms and in normal adult and fetal tissue. Immunochemistry. 11: 423 (1974).

6. Eveleigh, J. W.: Heterogeneity of carcinoembryonic antigen. Cancer Res., 34: 2122 (1974).
7. Gold. P.. and Freedman, S. O.: Demonstration of tumor-specific antigens in human colonic carcinomata by immunological tolerance and adsorption techniques. J. Exp. Med., 121: 439 (1965).

8. Gold, P., and Freedman. S. O.: Specific carcinoembryonic antigens of the human digestive system. J. Exp. Med., 122: 467 (1965).

9. Khoo, S. K.. Warner. N. L., Lie. J. T., and MacKay, I. R.: Carcinoembryonic antigen activity of tissue extracts: a quantitative study of malignant and benign neoplasms, cirrhotic liver, normal adult and fetal organs. Int. J. Cancer, 11 : 681 (1973)

10. Lowry, O. H., Rosebrough. N. J.. Farr, A. L., and Randall. R. J.: Protein measurement with the folin phenol reagent. J. Biol. Chem. 193: 265 (1951).

11. Mach. J. P.. and Pusztaszeri. G.: Carcinoembryonic antigen (CEA): demonstration of a partial identity between CEA and a normal glycoprotein. Immunochemistry, 9: 1031 (1972).

12. MacSween, J. M.. Warner, N. L., and MacKay, I. R.: The detection of carcinoembryonic antigen in whole serum from patients with malignant and nonmalignant disease. Clin. Immunol. Immunopathol.. l: 330 (1973).

13. Martin. F.. and Devant, J.: Carcinoembryonic antigen in normal human saliva J. Natl. Cancer Inst.. 50: 1375 (1973).

14. Molnar. I. G., Vandevoorde, J. P., and Gitnik. G. L.: CEA levels in fluids bathing gastrointestinal tumors. Gastroenterology. 70: 513 (1976).

15. Rule. A. H., and Goleski-Reilly. C.: Carcinoembryonic antigen (CEA): separation of CEA-reacting molecules from tumor, fetal gut, meconium and normal colon. Immunol. Commun., 2: 213 (1973).

16. Von Kleist. S.: Substances immunologically related to CEA. Ann. Immunol. 124C: 589 (1973)

17. Wilson. G. B., Burdash. N. M., Arnaud. P.. Monsher. M. T... and Fudenberg. H. H.: Carcinoembryonic antigen and cystic fibrosis protein in blood from cystic fibrosis homozygotes and heterozygote carriers. Scand. J. Immunol.. 5: 829 (1976).

18. Wu. J. T.. Herbst. J. J., and Bray. P. F.: Elevated plasma levels of carcinoembryonic antigen in cystic fibrosis. Pediatr. Res.. 10: 235 (1976).

19. The authors wish to thank Stuart Eastwood for excellent lechnical assistance and Pat Godin for secretarial assistance. This work was supported by the Medical Research Council of Canada.

20. Received for publication October 26. 1977

21. Accepted for publication April 10, 1979. 